\title{
Numerical Solution for Hybrid Fuzzy Differential Equation by Fifth Order Runge-Kutta Nystrom Method
}

\author{
Muthukumar Thangamuthu ${ }^{1 *}$ and Jayakumar Thippan ${ }^{2}$ \\ ${ }^{1}$ Department of Mathematics, Padmavani Arts and Science College for Women, Salem-636 011, Tamilnadu, India \\ ${ }^{2}$ Sri Ramakrishna Mission Vidyalaya College of Arts and Science, Coimbatore-641 020, Tamilnadu, India \\ * Corresponding author
}

\section{Article Info}

Keywords: Fuzzy differential equations, Hybrid systems, Runge-Kutta Nystrom method of order five

2010 AMS: $65 L x x$

Received: 13 June 2018

Accepted: 22 January 2019

Available online: 20 April 2019

\begin{abstract}
This study discusses a numerical methods for hybrid fuzzy differential equations by fifth order RK Nystrom Method for fuzzy differential equations. We prove the convergence result and give numerical examples to illustrate the theory.
\end{abstract}

\section{Introduction}

The topic of fuzzy differential equations(FDEs) has been rapidly growing in recent years. The concept of fuzzy derivative was first introduced by Chang and Zadeh [1] , it was followed up by Dubois and Prade [2] by using the extension principal in their approach. Other methods have been discussed by Puri and Ralescu [3] and Goetschel and Voxman [4]. Kandel and Byatt [5] applied the concept of fuzzy differential equation (FDE) to the analysis of fuzzy dynamical problems. The FDE and the initial value problem(Cauchy problem) were rigorously treated by Kaleva [6, 7], Seikkala [8], He and Yi [9], Kloeden [10] and by other researchers [11, 12]. Recently several authors has investigate hybrid FDEs $[13,14,15,16]$.

Hybrid systems are devoted to modeling, design, and validation of interactive systems of computer programs and continuous systems. These are, control systems that are capable of controlling complex systems which have discrete dynamics event as well as continuous time dynamics can be modeled by hybrid system. Hybrid system evolve in continuous time like differential systems but undergo fundamental changes in their governing equations at a sequence of discrete times. For analytical results on stability properties and comparison theorems we refer to $[3,8,17,18]$.

In this paper, we develop numerical methods for solving hybrid fuzzy differential equations by Runge-Kutta Nystrom method using the Seikkala derivative. In Section 2 we list some basic definitions for fuzzy valued functions. In Section 3 we review hybrid fuzzy differential systems. In Section 4 the Runge-Kutta Nystrom method of order five for solving hybrid fuzzy differential equations and a convergence theorem are discussed. Section 5 contains a some numerical examples to illustrate the theory.

\section{Preliminaries}

Denote by $E^{1}$ the set of all functions $u: R \rightarrow[0,1]$ such that (i) $v$ is normal, that is, there exist an $x_{0} \in R$ such that $v\left(x_{0}\right)=1$, (ii) $u$ is a fuzzy convex, that is, for $x, y \in R$ and $0 \leq \lambda \leq 1, v(\lambda x+(1-\lambda) y) \geq \min \{v(x), v(y)\}$, (iii) $v$ is upper semi continuous, and (iv) $[v]^{0} \equiv$ the closure of $\{x \in R: v(x)>0\}$ is compact. For $0<r \leq 1$, we define $[v]^{r}=\{x \in R: v(x) \geq r\}$. An example of a $v \in E^{1}$ is given by

$$
v(x)=\left\{\begin{array}{lc}
4 x-3, & \text { if } x \in(0.75,1], \\
-2 x+3, & \text { if } x \in(1,1.5), \\
0, & \text { if } x \notin(0.75,1.5) .
\end{array}\right.
$$




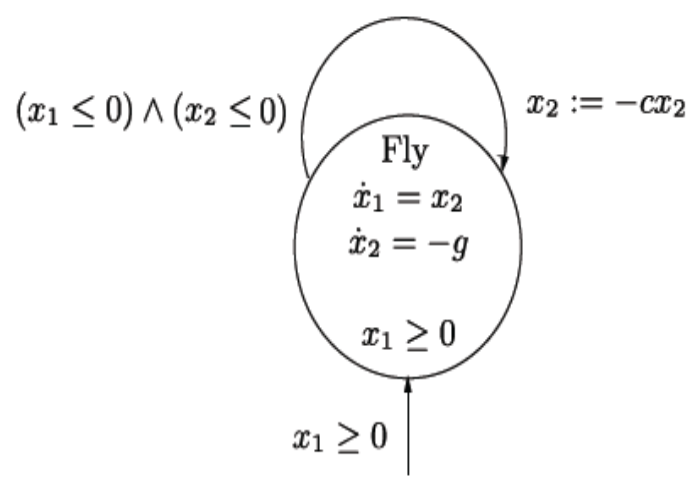

Figure 3.1: Bouncing ball.

The $r$-level sets of $u$ in (2.1) are given by

$$
[v]^{r}=[0.75+0.25 r, 1.5-0.5 r]
$$

For later purpose, we define $\hat{0} \in E^{1}$ as $\hat{0}(x)=1$ if $x=0$ and $\hat{0}(x)=0$ if $x \neq 0$.

Next we review the Seikkala derivative [8] of $x: I \rightarrow E^{1}$ where $I \subset R$ is an interval. If $[x(t)]^{r}=\left[\underline{x}^{r}(t), \bar{x}^{r}(t)\right]$ for all $t \in I$ and $r \in[0,1]$, then $\left[x^{\prime}(t)\right]^{r}=\left[\left(\underline{x}^{r}\right)^{\prime}(t),\left(\bar{x}^{r}\right)^{\prime}(t)\right]$ if $x^{\prime}(t) \in E^{1}$. Next consider the initial value problem(IVP)

$$
x^{\prime}(t)=g(t, x(t)), x(0)=x_{0}
$$

where $f:[0, \infty) \times R \rightarrow R$ is continuous. We would like to interpret (2.3) using the Seikkala derivative and $x_{0} \in E^{1}$. Let $\left[x_{0}\right]^{r}=\left[\underline{x}_{0}^{r}, \bar{x}_{0}^{r}\right]$ and $[x(t)]^{r}=\left[\underline{x}^{r}(t), \bar{x}^{r}(t)\right]$. By the Zadeh extension principle we get $g:[0, \infty) \times E^{1} \rightarrow E^{1}$ where

$$
[g(t, x)]^{r}=\left[\min \left\{g(t, v): v \in\left[\underline{x}^{r}(t), \bar{x}^{r}(t)\right]\right\}, \max \left\{g(t, v): v \in\left[\underline{x}^{r}(t), \bar{x}^{r}(t)\right]\right\}\right] .
$$

Then $x:[0, \infty) \rightarrow E^{1}$ is a solution of (2.3) using the Seikkala derivative and $x_{0} \in E^{1}$ if

$$
\begin{array}{ll}
\left(\underline{x}^{r}\right)^{\prime}(t)=\min \left\{g(t, v): v \in\left[\underline{x}^{r}(t), \bar{x}^{r}(t)\right]\right\}, & \underline{x}^{r}(0)=x_{0}^{r}, \\
\left(\bar{x}^{r}\right)^{\prime}(t)=\max \left\{g(t, v): v \in\left[\underline{x}^{r}(t), \bar{x}^{r}(t)\right]\right\}, & \bar{x}^{r}(0)=\bar{x}_{0}^{r},
\end{array}
$$

for all $t \in[0, \infty)$ and $r \in[0,1]$. Lastly consider an $g:[0, \infty) \times R \times R \rightarrow R$ which is continuous and the IVP

$$
\left\{\begin{array}{l}
x^{\prime}(t)=g(t, x(t), k) \\
x(0)=x_{0}
\end{array}\right.
$$

As in [19], to interpret (2.4) using the Seikkala derivative and $x_{0}, k \in E^{1}$, by the Zadeh extension principle we use $g:[0, \infty) \times E^{1} \times E^{1} \rightarrow E^{1}$ where

$$
\begin{aligned}
{[g(t, x, k)]^{r}=} & {\left[\min \left\{g\left(t, v, v_{k}\right): v \in\left[\underline{x}^{r}(t), \bar{x}^{r}(t)\right], \quad v_{k} \in\left[\underline{k}^{r}, \bar{k}^{r}\right]\right\},\right.} \\
& \left.\max \left\{g\left(t, v, v_{k}\right): v \in\left[\underline{x}^{r}(t), \bar{x}^{r}(t)\right], \quad v_{k} \in\left[\underline{k}^{r}, \bar{k}^{r}\right]\right\}\right],
\end{aligned}
$$

where $k^{r}=\left[\underline{k}^{r}, \bar{k}^{r}\right]$. Then $x:[0, \infty) \rightarrow E^{1}$ is a solution of (2.4) using the Seikkala derivative and $x_{0}, k \in E^{1}$ if

$$
\begin{aligned}
& \left(\underline{x}^{r}\right)^{\prime}(t)=\min \left\{g\left(t, v, v_{k}\right): v \in\left[\underline{x}^{r}(t), \bar{x}^{r}(t)\right], v_{k} \in\left[\underline{k}^{r}, \bar{k}^{r}\right]\right\}, \quad \underline{x}^{r}(0)=\underline{x}_{0}^{r}, \\
& \left(\bar{x}^{r}\right)^{\prime}(t)=\max \left\{g\left(t, v, v_{k}\right): v \in\left[\underline{x}^{r}(t), \bar{x}^{r}(t)\right], v_{k} \in\left[\underline{k}^{r}, \bar{k}^{r}\right]\right\}, \quad \bar{x}^{r}(0)=\bar{x}_{0}^{r},
\end{aligned}
$$

for all $t \in[0, \infty)$ and $r \in[0,1]$.

\section{The hybrid fuzzy differential systems}

Hybrid systems have been used to model several cyber-physical systems, including physical systems with impact, logic-dynamic controllers, and even Internet congestion.

A canonical example of a hybrid system is the bouncing ball, the physical system with impact. Here, the ball (thought of as a point-mass) is dropped from an initial height and bounces off the ground, dissipating its energy with each bounce. The ball exhibits continuous dynamics between each bounce; however, as the ball impacts the ground, its velocity undergoes a discrete change modeled after an inelastic collision. A mathematical description of the bouncing ball follows. Let $x_{1}$ be the height of the ball and $x_{2}$ be the velocity of the ball. A hybrid system describing the ball is as follows:

When $x \in C=\left\{x_{1} \geq 0\right\}$, flow is governed by $\dot{x}_{1}=x_{2}, \dot{x_{2}}=-g$, where $\mathrm{g}$ is the acceleration due to gravity. These equations state that when the ball is above ground, it is being drawn to the ground by gravity.

When $x \in D=\left\{x_{1}=0\right\}$, jumps are governed by $x_{1}^{+}=x_{1}, x_{2}^{+}=-\gamma x_{2}$, where $0<\gamma<1$ is a dissipation factor. This is saying that when the height of the ball is zero (it has impacted the ground), its velocity is reversed and decreased by a factor of $\gamma$. Effectively, this describes the nature of the inelastic collision. 


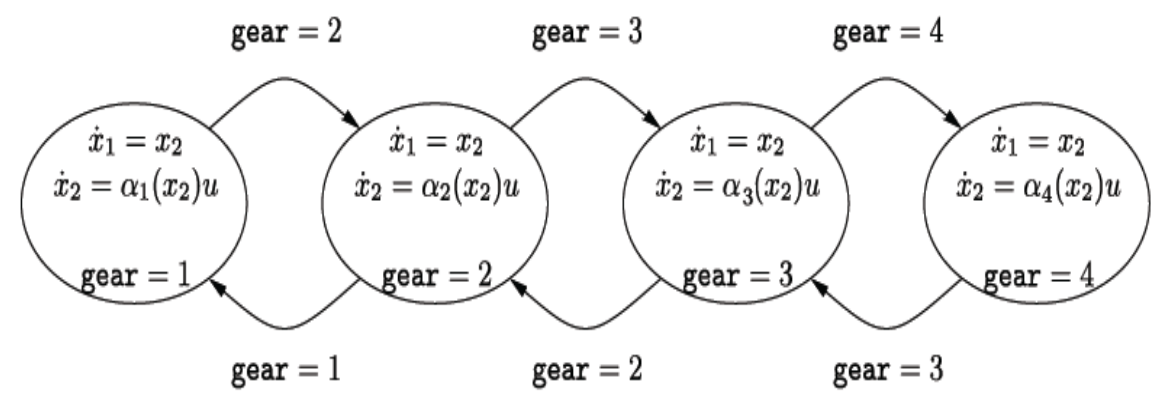

Figure 3.2: A hybrid system modeling a car with four gears.

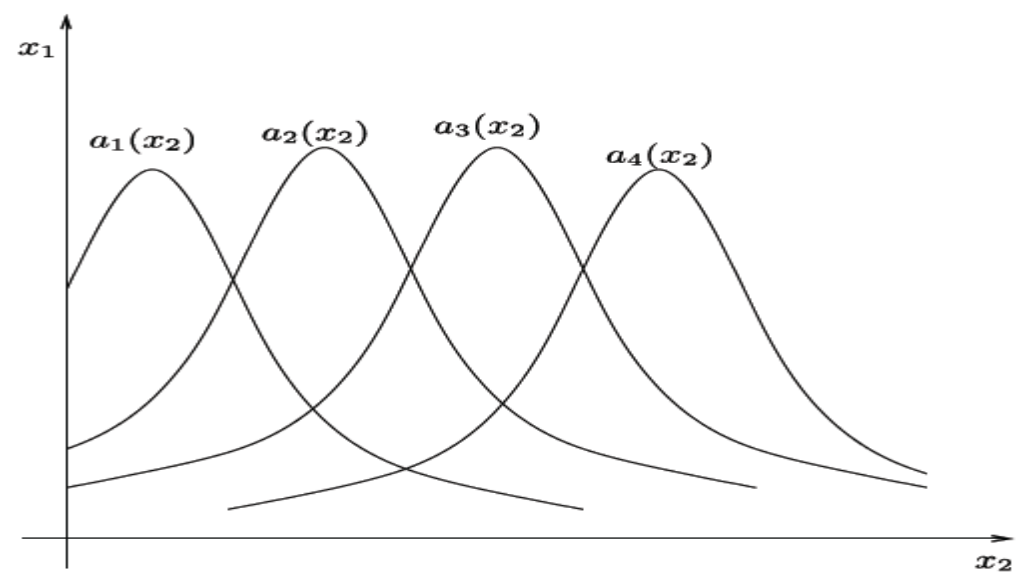

Figure 3.3: The efficiency functions of the different gears

\section{Car Gear shift:}

The gear shift example describes a control design problem where both the continuous and the discrete controls need to be determined. Figure 3.2 shows a model of a car with a gear box having four gears. The longitudinal position of the car along the road is denoted by $x_{1}$ and its velocity by $x_{2}$ (lateral dynamics are ignored). The model has two control signals; the gear denoted gear $\in\{1, \ldots, 4\}$ and the throttle position denoted $u \in\left[u_{\min }, u_{\max }\right]$. Gear shifting is necessary because little power can be ignored by the engine at very low or very high engine speed. The function $\alpha_{i}$ represents the efficiency of the gear i. Typical shapes of the function $\alpha_{i}$ are shown in the Figure 3.3.

How many real valued continuous states does this model have? How many discrete states?

Several interesting control problems can be posed for this simple car model. For example, what is the optimal control strategy to drive from $(a, 0)$ to $(b, 0)$ in a minimum time? The problem is not trivial if we include the reasonable assumption that each gear shift takes a certain amount of time. The optimal control of hybrid system, may be derived using the theory of optimal control of hybrid systems.

Consider the hybrid fuzzy differential system

$$
\left\{\begin{array}{l}
x^{\prime}(t)=g\left(t, x(t), \lambda_{k}\left(x_{k}\right)\right), \quad t \in\left[t_{k}, t_{k+1}\right] \\
x\left(t_{k}\right)=x_{k}
\end{array}\right.
$$

where' denotes Seikkala differentiation, $0 \leq t_{0}<t_{1}<\cdots<t_{k}<\cdots, t_{k} \rightarrow \infty$, $g \in C\left[R^{+} \times E^{1} \times E^{1}, E^{1}\right], \lambda_{k} \in C\left[E^{1}, E^{1}\right]$. To be specific the system look like

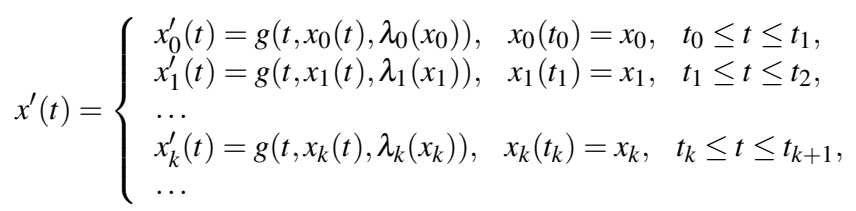

Discuss the existence and uniqueness of solution of (3.1) hold for each $\left[t_{k}, t_{k+1}\right]$, by the solution of (2.3) we mean the following function:

$$
x(t)=x\left(t, t_{0}, x_{0}\right)= \begin{cases}x_{0}(t), & t_{0} \leq t \leq t_{1} \\ x_{1}(t), & t_{1} \leq t \leq t_{2} \\ \cdots & \\ x_{k}(t), & t_{k} \leq t \leq t_{k+1} \\ \cdots & \end{cases}
$$

We note that the solution of (3.1) are piecewise differentiable in each interval for $t \in\left[t_{k}, t_{k+1}\right]$ for a fixed $x_{k} \in E^{1}$ and $k=0,1,2, \ldots$ 
Using a representation of fuzzy numbers studied by Goestschel and Voxman [4] and Wu and Ma [18], we may represent $x \in E^{1}$ by a pair of fuctions $(\underline{x}(r), \bar{x}(r)), 0 \leq r \leq 1$, such that (i) $\underline{x}(r)$ is bounded, left continuous, and nondecreasing, (ii) $\bar{x}(r)$ is bounded, left continuous, and nonincreasing, and (iii) $\underline{x}(r) \leq \bar{x}(r), 0 \leq r \leq 1$. For example , $v \in E^{1}$ given in $((2.1)$ is represented by $(\underline{v}(r), \bar{v}(r))=$ $(0.75+0.25 r, 1.5-0.5 r), 0 \leq r \leq 1$, which is similar to $[v]^{r}$ given by $(2.2)$.

Therefore we may replace (3.1) by an equivalent system

$$
\begin{cases}\underline{x}^{\prime}(t)=\underline{g}\left(t, x, \lambda_{k}\left(x_{k}\right)\right) \equiv F_{k}(t, \underline{x}, \bar{x}), & \underline{x}\left(t_{k}\right)=\underline{x}_{k}, \\ \bar{x}^{\prime}(t)=\bar{g}\left(t, x, \lambda_{k}\left(x_{k}\right)\right) \equiv G_{k}(t, \underline{x}, \bar{x}), & \bar{x}\left(t_{k}\right)=\bar{x}_{k},\end{cases}
$$

which possesses a unique solution $(\underline{x}, \bar{x})$ which is a fuzzy function. That is for each $t$, the pair $[\underline{x}(t ; r), \bar{x}(t ; r)]$ is a fuzzy number, where $\underline{x}(t ; r), \bar{x}(t ; r)$ are respectively the solutions of the parametric form given by

$$
\begin{cases}\underline{x}^{\prime}(t ; r)=F_{k}[t, \underline{x}(t ; r), \bar{x}(t ; r)], & \underline{x}\left(t_{k} ; r\right)=\underline{x}_{k}(r), \\ \bar{x}^{\prime}(t ; r)=G_{k}[t, \underline{x}(t ; r), \bar{x}(t ; r)], & \bar{x}\left(t_{k} ; r\right)=\bar{x}_{k}(r),\end{cases}
$$

for $r \in[0,1]$.

\section{The Runge-Kutta Nystrom method}

In this section, for a hybrid fuzzy differential equation (3.1) we develop the fifth order Runge-Kutta Nystrom method when $f$ and $\lambda_{k}$ in (2.3) can be obtained via the Zadeh extension principle from $f \in C\left[R^{+} \times R \times R, R\right]$ and $\lambda_{k} \in C[R, R]$ (since we are using the Seikkala derivative). We assume that the existence and uniqueness of solutions of (3.1) hold for each $\left[t_{k}, t_{k+1}\right]$.

For a fixed $r$, to integrate the system in (3.3) in $\left[t_{0}, t_{1}\right],\left[t_{1}, t_{2}\right], \ldots,\left[t_{k}, t_{k+1}\right], \ldots$, we replace each interval by a set of $N_{k}+1$ discrete equally spaced grid points (including the end points) at which exact solution $x(t ; r)=(\underline{x}(t ; r), \bar{x}(t ; r))$ is approximated by some $\left(\underline{y}_{k}(t ; r), \bar{y}_{k}(t ; r)\right)$. For each the chosen grid points on $\left[t_{k}, t_{k+1}\right]$ at $t_{k, n}=t_{k}+n h_{k}, h_{k}=\frac{t_{k+1}-t_{k}}{N_{k}}, 0 \leq n \leq N_{k}$, Let $\left(\underline{Y}_{k}(t ; r), \bar{Y}_{k}(t ; r)\right) \equiv(\underline{x}(t ; r), \bar{x}(t ; r)),\left(\underline{Y}_{k}(t ; r), \bar{Y}_{k}(t ; r)\right)$ and $\left(\underline{y}_{k}(t ; r), \bar{y}_{k}(t ; r)\right)$ may be denoted respectively by $\left(\underline{Y}_{k, n}(r), \bar{Y}_{k, n}(r)\right)$ and $\left(\underline{y}_{k, n}(r), \bar{y}_{k, n}(r)\right)$. We allow the $N_{k}$ 's to vary over the $\left[t_{k}, t_{k+1}\right]$ 's so that the $h_{k}$ 's may be comparable.

The Runge-Kutta Nystrom method is a fifth order approximation of $\underline{Y}_{k}^{\prime}(t ; r)$ and $\bar{Y}_{k}^{\prime}(t ; r)$. To develop the Runge-Kutta Nystrom method for (2.3), and define

$$
\begin{aligned}
y_{k, n+1}(r)-\underline{y}_{k, n}(r) & =\sum_{i=1}^{6} w_{i} \underline{k}_{i}\left(t_{k, n} ; y_{k, n}(r)\right), \\
\bar{y}_{k, n+1}(r)-\bar{y}_{k, n}(r) & =\sum_{i=1}^{6} w_{i} \bar{k}_{i}\left(t_{k, n} ; y_{k, n}(r)\right),
\end{aligned}
$$

where $w_{1}, w_{2}, w_{3}, w_{4}, w_{5}$ and $w_{6}$ are constants and

$$
\begin{aligned}
\underline{k}_{1}\left(t_{k, n} ; y_{k, n}(r)\right)= & \min \left\{h_{k} g\left(t_{k, n}, v, \lambda_{k}\left(v_{k}\right)\right) \mid\right. \\
v & \left.\in\left[\underline{y}_{k, n}(r), \bar{y}_{k, n}(r)\right], v_{k} \in\left[\underline{y}_{k, 0}(r), \bar{y}_{k, 0}(r)\right]\right\}, \\
\bar{k}_{1}\left(t_{k, n} ; y_{k, n}(r)\right)= & \max \left\{h_{k} g\left(t_{k, n}, v, \lambda_{k}\left(v_{k}\right)\right) \mid\right. \\
& \left.v \in\left[\underline{y}_{k, n}(r), \bar{y}_{k, n}(r)\right], v_{k} \in\left[\underline{y}_{k, 0}(r), \bar{y}_{k, 0}(r)\right]\right\}, \\
\underline{k}_{2}\left(t_{k, n} ; y_{k, n}(r)\right)= & \min \left\{h_{k} g\left(t_{k, n}+\frac{1}{3} h_{k}, v, \lambda_{k}\left(v_{k}\right)\right) \mid\right. \\
\bar{k}_{2}\left(t_{k, n} ; y_{k, n}(r)\right)= & \max \left\{\underline{h}_{k} g\left(t_{k, n}+\frac{1}{3} h_{k}, v, \lambda_{k}\left(v_{k}\right)\right) \mid\right. \\
v & \left.v\left[\underline{z}_{k_{1}}\left(t_{k, n}, y_{k, n}(r)\right), \bar{z}_{k_{1}}\left(t_{k, n}, y_{k, n}(r)\right)\right], v_{k} \in\left[\underline{y}_{k, 0}(r), \bar{y}_{k, 0}(r)\right]\right\}, \\
\underline{k}_{3}\left(t_{k, n} ; y_{k, n}(r)\right)= & \min \left\{h_{k} g\left(t_{k, n}+\frac{2}{5} h_{k}, v, \lambda_{k}\left(v_{k}\right)\right) \mid\right. \\
v & \left.v\left[\underline{z}_{k_{2}}\left(t_{k, n}, y_{k, n}(r)\right), \bar{z}_{k_{2}}\left(t_{k, n}, y_{k, n}(r)\right)\right], v_{k} \in\left[\underline{y}_{k, 0}(r), \bar{y}_{k, 0}(r)\right]\right\},
\end{aligned}
$$




$$
\begin{aligned}
& \bar{k}_{3}\left(t_{k, n} ; y_{k, n}(r)\right)=\max \left\{h_{k} g\left(t_{k, n}+\frac{2}{5} h_{k}, v, \lambda_{k}\left(v_{k}\right)\right) \mid\right. \\
& \left.v \in\left[\underline{z}_{k_{2}}\left(t_{k, n}, y_{k, n}(r)\right), \bar{z}_{k_{2}}\left(t_{k, n}, y_{k, n}(r)\right)\right], v_{k} \in\left[\underline{y}_{k, 0}(r), \bar{y}_{k, 0}(r)\right]\right\}, \\
& \underline{k}_{4}\left(t_{k, n} ; y_{k, n}(r)\right)=\min \left\{h_{k} g\left(t_{k, n}+h_{k}, v, \lambda_{k}\left(v_{k}\right)\right) \mid\right. \\
& \left.v \in\left[\underline{z}_{k_{3}}\left(t_{k, n}, y_{k, n}(r)\right), \bar{z}_{k_{3}}\left(t_{k, n}, y_{k, n}(r)\right)\right], v_{k} \in\left[\underline{y}_{k, 0}(r), \bar{y}_{k, 0}(r)\right]\right\}, \\
& \bar{k}_{4}\left(t_{k, n} ; y_{k, n}(r)\right)=\max \left\{h_{k} g\left(t_{k, n}+h_{k}, v, \lambda_{k}\left(v_{k}\right)\right) \mid\right. \\
& \left.v \in\left[\underline{z}_{k_{3}}\left(t_{k, n}, y_{k, n}(r)\right), \bar{z}_{k_{3}}\left(t_{k, n}, y_{k, n}(r)\right)\right], v_{k} \in\left[\underline{y}_{k, 0}(r), \bar{y}_{k, 0}(r)\right]\right\}, \\
& \underline{k}_{5}\left(t_{k, n} ; y_{k, n}(r)\right)=\min \left\{h_{k} g\left(t_{k, n}+\frac{2}{3} h_{k}, v, \lambda_{k}\left(v_{k}\right)\right) \mid\right. \\
& \left.v \in\left[\underline{z}_{k_{4}}\left(t_{k, n}, y_{k, n}(r)\right), \bar{z}_{k_{4}}\left(t_{k, n}, y_{k, n}(r)\right)\right], v_{k} \in\left[\underline{y}_{k, 0}(r), \bar{y}_{k, 0}(r)\right]\right\}, \\
& \bar{k}_{5}\left(t_{k, n} ; y_{k, n}(r)\right)=\max \left\{h_{k} g\left(t_{k, n}+\frac{2}{3} h_{k}, v, \lambda_{k}\left(v_{k}\right)\right) \mid\right. \\
& \left.v \in\left[\underline{z}_{k_{4}}\left(t_{k, n}, y_{k, n}(r)\right), \bar{z}_{k_{4}}\left(t_{k, n}, y_{k, n}(r)\right)\right], v_{k} \in\left[\underline{y}_{k, 0}(r), \bar{y}_{k, 0}(r)\right]\right\}, \\
& \underline{k}_{6}\left(t_{k, n} ; y_{k, n}(r)\right)=\min \left\{h_{k} g\left(t_{k, n}+\frac{4}{5} h_{k}, v, \lambda_{k}\left(v_{k}\right)\right) \mid\right. \\
& \left.v \in\left[\underline{z}_{k_{5}}\left(t_{k, n}, y_{k, n}(r)\right), \bar{z}_{k_{5}}\left(t_{k, n}, y_{k, n}(r)\right)\right], v_{k} \in\left[\underline{y}_{k, 0}(r), \bar{y}_{k, 0}(r)\right]\right\}, \\
& \bar{k}_{6}\left(t_{k, n} ; y_{k, n}(r)\right)=\max \left\{h_{k} g\left(t_{k, n}+\frac{4}{5} h_{k}, v, \lambda_{k}\left(v_{k}\right)\right) \mid\right. \\
& \left.v \in\left[\underline{z}_{k_{5}}\left(t_{k, n}, y_{k, n}(r)\right), \bar{z}_{k_{5}}\left(t_{k, n}, y_{k, n}(r)\right)\right], v_{k} \in\left[\underline{y}_{k, 0}(r), \bar{y}_{k, 0}(r)\right]\right\}, \\
& \underline{z}_{k_{1}}\left(t_{k, n}, y_{k, n}(r)\right)=\underline{y}_{k, n}(r)+\frac{1}{3} \underline{k}_{1}\left(t_{k, n}, y_{k, n}(r)\right), \\
& \bar{z}_{k_{1}}\left(t_{k, n}, y_{k, n}(r)\right)=\bar{y}_{k, n}(r)+\frac{1}{3} \bar{k}_{1}\left(t_{k, n}, y_{k, n}(r)\right), \\
& \underline{z}_{k_{2}}\left(t_{k, n}, y_{k, n}(r)\right)=\underline{y}_{k, n}(r)+\frac{4}{25} \underline{k}_{1}\left(t_{k, n}, y_{k, n}(r)\right)+\frac{6}{25} \underline{k}_{2}\left(t_{k, n}, y_{k, n}(r)\right), \\
& \bar{z}_{k_{2}}\left(t_{k, n}, y_{k, n}(r)\right)=\bar{y}_{k, n}(r)+\frac{4}{25} \bar{k}_{1}\left(t_{k, n}, y_{k, n}(r)\right)+\frac{6}{25} \bar{k}_{2}\left(t_{k, n}, y_{k, n}(r)\right) \text {, } \\
& \underline{z}_{k_{3}}\left(t_{k, n}, y_{k, n}(r)\right)=\underline{y}_{k, n}(r)+\frac{1}{4} \underline{k}_{1}\left(t_{k, n}, y_{k, n}(r)\right) \\
& -\frac{12}{4} \underline{k}_{2}\left(t_{k, n}, y_{k, n}(r)\right)+\frac{15}{4} \underline{k}_{3}\left(t_{k, n}, y_{k, n}(r)\right), \\
& \bar{z}_{k_{3}}\left(t_{k, n}, y_{k, n}(r)\right)=\bar{y}_{k, n}(r)+\frac{1}{4} \bar{k}_{1}\left(t_{k, n}, y_{k, n}(r)\right) \\
& -\frac{12}{4} \bar{k}_{2}\left(t_{k, n}, y_{k, n}(r)\right)+\frac{15}{4} \bar{k}_{3}\left(t_{k, n}, y_{k, n}(r)\right) \text {, } \\
& \underline{z}_{k_{4}}\left(t_{k, n}, y_{k, n}(r)\right)=\underline{y}_{k, n}(r)+\frac{6}{81} \underline{k}_{1}\left(t_{k, n}, y_{k, n}(r)\right) \\
& +\frac{90}{81} \underline{k}_{2}\left(t_{k, n}, y_{k, n}(r)\right)-\frac{50}{81} \underline{k}_{3}\left(t_{k, n}, y_{k, n}(r)\right)+\frac{8}{81} \underline{k}_{4}\left(t_{k, n} ; y_{k, n}(r)\right), \\
& \bar{z}_{k_{4}}\left(t_{k, n}, y_{k, n}(r)\right)=\bar{y}_{k, n}(r)+\frac{6}{81} \bar{k}_{1}\left(t_{k, n}, y_{k, n}(r)\right) \\
& +\frac{90}{81} \bar{k}_{2}\left(t_{k, n}, y_{k, n}(r)\right)-\frac{50}{81} \bar{k}_{3}\left(t_{k, n}, y_{k, n}(r)\right)+\frac{8}{81} \bar{k}_{4}\left(t_{k, n} ; y_{k, n}(r)\right), \\
& \underline{z}_{k_{5}}\left(t_{k, n}, y_{k, n}(r)\right)=\underline{y}_{k, n}(r)+\frac{6}{75} \underline{k}_{1}\left(t_{k, n}, y_{k, n}(r)\right) \\
& +\frac{36}{75} \underline{k}_{2}\left(t_{k, n}, y_{k, n}(r)\right)+\frac{10}{75} \underline{k}_{3}\left(t_{k, n}, y_{k, n}(r)\right)+\frac{8}{75} \underline{k}_{4}\left(t_{k, n} ; y_{k, n}(r)\right), \\
& \bar{z}_{k_{5}}\left(t_{k, n}, y_{k, n}(r)\right)=\bar{y}_{k, n}(r)+\frac{6}{75} \bar{k}_{1}\left(t_{k, n}, y_{k, n}(r)\right) \\
& +\frac{36}{75} \bar{k}_{2}\left(t_{k, n}, y_{k, n}(r)\right)+\frac{10}{75} \bar{k}_{3}\left(t_{k, n}, y_{k, n}(r)\right)+\frac{8}{75} \bar{k}_{4}\left(t_{k, n} ; y_{k, n}(r)\right),
\end{aligned}
$$


Next we define

$$
\begin{aligned}
S_{k}\left[t_{k, n}, \underline{y}_{k, n}(r), \bar{y}_{k, n}(r)\right]= & 23 \underline{k}_{1}\left(t_{k, n}, y_{k, n}(r)+125 \underline{k}_{3}\left(t_{k, n}, y_{k, n}(r)\right)\right. \\
& -81 \underline{k}_{5}\left(t_{k, n}, y_{k, n}(r)\right)+125 \underline{k}_{6}\left(t_{k, n}, y_{k, n}(r)\right), \\
T_{k}\left[t_{k, n}, \underline{y}_{k, n}(r), \bar{y}_{k, n}(r)\right]= & 23 \bar{k}_{1}\left(t_{k, n}, y_{k, n}(r)+125 \bar{k}_{3}\left(t_{k, n}, y_{k, n}(r)\right)\right. \\
& -81 \bar{k}_{5}\left(t_{k, n}, y_{k, n}(r)\right)+125 \bar{k}_{6}\left(t_{k, n}, y_{k, n}(r)\right)
\end{aligned}
$$

The exact solution at $t_{k, n+1}$ is given by

$$
\left\{\begin{array}{c}
\underline{Y}_{k, n+1}(r) \approx \underline{Y}_{k, n}(r)+\frac{1}{192} S_{k}\left[t_{k, n}, \underline{Y}_{k, n}(r), \bar{Y}_{k, n}(r)\right], \\
\bar{Y}_{k, n+1}(r) \approx \bar{Y}_{k, n}(r)+\frac{1}{192} T_{k}\left[t_{k, n}, \bar{Y}_{k, n}(r), \bar{Y}_{k, n}(r)\right] .
\end{array}\right.
$$

The approximate solution is given by

$$
\left\{\begin{array}{c}
\underline{y}_{k, n+1}(r) \approx \underline{y}_{k, n}(r)+\frac{1}{192} S_{k}\left[t_{k, n}, \underline{y}_{k, n}(r), \bar{y}_{k, n}(r)\right], \\
\bar{y}_{k, n+1}(r) \approx \bar{y}_{k, n}(r)+\frac{1}{192} T_{k}\left[t_{k, n}, \bar{y}_{k, n}(r), \bar{y}_{k, n}(r)\right] .
\end{array}\right.
$$

Lemma 4.1. Suppose $k \in Z^{+}, \varepsilon_{k}>0, r \in[0,1]$, and $h_{k}<1$ are fixed. Let $\left\{Z_{k, n}(r)\right\}_{n=0}^{N_{k}}$ be the fifth order $R$-K Nystrom method approximation with $N=N_{k}$ to the fuzzy IVP:

$$
\left\{\begin{array}{l}
x^{\prime}(t)=f\left(t, x(t), \lambda_{k}\left(x_{k}\right)\right), \quad t \in\left[t_{k}, t_{k+1}\right] \\
x\left(t_{k}\right)=x_{k}
\end{array}\right.
$$

If $\left\{y_{k, n}(r)\right\}_{n=0}^{N_{k}}$ denotes the result of (3.3) from some $y_{k, 0}(r)$, then there exists $\delta_{k}>0$ such that $\left|\underline{z}_{k, 0}(r)-\underline{y}_{k, 0}(r)\right|<\delta_{k},\left|\bar{z}_{k, 0}(r)-\bar{y}_{k, 0}(r)\right|<\delta_{k}$ imply $\left|\underline{z}_{k, 0}(r)-\underline{y}_{k, 0}(r)\right|<\varepsilon_{k},\left|\bar{z}_{k, 0}(r)-\bar{y}_{k, 0}(r)\right|<\varepsilon_{k}$.

Theorem 4.2. Consider the systems (3.2) and (4.1). For a fixed $k \in Z^{+}$and $r \in[0,1]$,

$$
\begin{array}{r}
\lim _{h_{0}, \ldots, h_{k} \rightarrow 0} \underline{y}_{k, N_{k}}(r)=\underline{x}\left(t_{k+1} ; r\right), \\
\lim _{h_{0}, \ldots, h_{k} \rightarrow 0} \bar{y}_{k, N_{k}}(r)=\bar{x}\left(t_{k+1} ; r\right) .
\end{array}
$$

\section{Numerical examples}

Consider the fuzzy differential equation

$$
x^{\prime}(t)=x(t), x(0 ; r)=[0.75+0.25 r, 1.125-0.125 r], 0 \leq r \leq 1 .
$$

By the fifth order Runge Kutta Nystorm method with $\mathrm{N}=10$

$$
y(1.0 ; r)=(0.75+0.25 r)\left(c_{0,1}\right)^{10},(1.125-0.125 r)\left(c_{0,1}\right)^{10},
$$

where $y(t ; r)$ denotes an approximate solution of (5.1). Since the exact solution of (5.1) is $x(t ; r)=\left[(0.75+0.25 r) e^{t},(1.125-0.125 r) e^{t}\right], 0 \leq$ $r \leq 1$, we see that $x(1 ; r)=[(0.75+0.25 r) e,(1.125-0.125 r) e], 0 \leq r \leq 1$, which compares well with (5.2). By the fifth order Runge Kutta Nystorm method with $N=10$,

$$
y(1.0 ; r)=\left[(0.75+0.25 r)\left(c_{0,1}\right)^{10},(1.125-0.125 r)\left(c_{0,1}\right)^{10}\right], 0 \leq r \leq 1,
$$

where $c_{0,1}=1+h+\frac{(h)^{2}}{2}+\frac{(h)^{3}}{6}+\frac{(h)^{4}}{24}+\frac{(h)^{5}}{120}$.

Example 5.1. Next consider the following hybrid fuzzy IVP,

$$
\left\{\begin{array}{l}
x^{\prime}(t)=x(t)+m(t) \lambda_{k} x\left(t_{k}\right), t \in\left[t_{k}, t_{k+1}\right], t_{k}=k, \quad k=0,1,2,3, \ldots, \\
x(t ; r)=\left[(0.75+0.25 r) e^{t},(1.125-0.125 r) e^{t}\right], 0 \leq r \leq 1,
\end{array}\right.
$$

where

$$
m(t)= \begin{cases}2(t(\bmod 1)) & \text { if } t(\bmod 1) \leq 0.5 \\ 2(1-t(\bmod 1) & \text { if } t(\bmod 1)>0.5\end{cases}
$$




$$
\lambda_{k}(\mu)= \begin{cases}\hat{0}, & \text { if } k=0 \\ \mu, & \text { if } k \in\{1,2, \ldots\}\end{cases}
$$

The hybrid fuzzy IVP (5.4) is equivalent to the following systems of fuzzy IVPs:

$$
\left\{\begin{array}{l}
x_{0}^{\prime}(t)=x_{0}(t), \quad t \in[0,1], \\
x_{0}(0 ; r)=[(0.75+0.25 r) e,(1.125-0.125 r) e], \quad 0 \leq r \leq 1 \\
x_{i}^{\prime}(t)=x_{i}(t)+m(t) x_{i-1}(t), t \in\left[t_{i}, t_{i+1}\right], x_{i}(t)=x_{i-1}\left(t_{i}\right), i=1,2, \ldots
\end{array}\right.
$$

In (5.4), $x(t)+m(t) \lambda_{k}\left(x\left(t_{k}\right)\right.$ is continous function of $t, x$ and $\lambda_{k}\left(x\left(t_{k}\right)\right.$. Therefore by Example 6.1 of Kaleva [6], for each $k=0,1,2, \ldots$, the fuzzy IVP

$$
\left\{\begin{array}{l}
x^{\prime}(t)=x(t)+m(t) \lambda_{k}\left(x\left(t_{k}\right)\right), \quad t \in\left[t_{k}, t_{k+1}\right], t_{k}=k, \\
x\left(t_{k}\right)=x_{t_{k}},
\end{array}\right.
$$

has a unique solution on $\left[t_{k}, t_{k+1}\right]$. To numerically solve the hybrid fuzzy IVP (5.4) we will apply the Runge-Kutta method of order five for hybrid fuzzy differential equation with $N=10$ to obtain $y_{1,2}(r)$ approximating $x(2.0 ; r)$. Let $f:[0, \infty) \times R \times R \rightarrow R$ be given by

$$
f\left(t, x, \lambda_{k}\left(x\left(t_{k}\right)\right)\right)=x(t)+m(t) \lambda_{k}\left(x\left(t_{k}\right)\right), t_{k}=k, k=0,1,2, \ldots,
$$

where $\lambda_{k}: R \rightarrow R$ is given by

$$
\lambda_{k}(x)= \begin{cases}0, & \text { if } k=0 \\ x, & \text { if } k \in\{1,2, \ldots\}\end{cases}
$$

By Example 1 of [19], (5.1) gives

$$
y_{1,0}(r)=\left[(0.75+0.25 r)\left(c_{0,1}\right)^{10},(1.125-0.125 r)\left(c_{0,1}\right)^{10}\right] .
$$

Next suppose $k=1$ and $n=0$. Then

$$
\begin{aligned}
& \underline{y}_{1,1}(r)=\underline{y}_{1,0}(r)+\frac{1}{192} S_{1}\left[1.0, \underline{y}_{1,0}(r), \bar{y}_{1,0}(r)\right], \\
& \bar{y}_{1,1}(r)=\bar{y}_{1,0}(r)+\frac{1}{192} T_{1}\left[1.0, \underline{y}_{1,0}(r), \bar{y}_{1,0}(r)\right] .
\end{aligned}
$$

To obtain $y_{1,1}(r), i=1,2,3,4,5$

$$
\begin{array}{r}
\underline{y}\left(1+\frac{i}{10} ; r\right)=\underline{y}\left(1+\frac{i-1}{10} ; r\right) c_{0,1}+\left[\frac{2 i-1}{100}+\frac{3 i-2}{3000}+\frac{4 i-3}{120000}+\frac{5 i-4}{6000000}\right. \\
\left.+\frac{i-1}{60000000}\right] \underline{y}(1.0 ; r), \\
\bar{y}\left(1+\frac{i}{10} ; r\right)=\bar{y}\left(1+\frac{i-1}{10} ; r\right) c_{0,1}+\left[\frac{2 i-1}{100}+\frac{3 i-2}{3000}+\frac{4 i-3}{120000}+\frac{5 i-4}{6000000}\right. \\
\left.+\frac{i-1}{60000000}\right] \bar{y}(1.0 ; r),
\end{array}
$$

Then for $i=6,7,8,9,10$

$$
\begin{array}{r}
\underline{y}\left(1+\frac{i}{10} ; r\right)=\underline{y}\left(1+\frac{i-1}{10} ; r\right) c_{0,1}+\left[\frac{1}{5}-\left(\frac{2 i-2}{100}+\frac{i-1}{1000}+\frac{i-1}{30000}+\frac{i-1}{1200000}\right.\right. \\
\left.\left.+\frac{i-1}{60000000}\right)\right] \underline{y}(1.0 ; r), \\
\bar{y}\left(1+\frac{i}{10} ; r\right)=\bar{y}\left(1+\frac{i-1}{10} ; r\right) c_{0,1}+\left[\frac{1}{5}-\left(\frac{2 i-2}{100}+\frac{i-1}{1000}+\frac{i-1}{30000}+\frac{i-1}{1200000}\right.\right. \\
\left.\left.+\frac{i-1}{60000000}\right)\right] \bar{y}(1.0 ; r)
\end{array}
$$

Let

$$
\begin{array}{r}
c_{2,0}=\left(c_{0,1}\right)^{10}+\sum_{k=1}^{5}\left(c_{0,1}\right)^{10-k}\left[\frac{2 k-1}{100}+\frac{3 k-2}{3000}+\frac{4 k-3}{120000}+\frac{5 k-4}{6000000}+\frac{k-1}{60000000}\right] \\
+\sum_{k=6}^{10}\left(c_{0,1}\right)^{10-k}\left[\frac{1}{5}-\left(\frac{2 k-2}{100}+\frac{k-1}{1000}+\frac{k-1}{30000}+\frac{k-1}{1200000}+\frac{k-1}{60000000}\right)\right] .
\end{array}
$$




\begin{tabular}{|c|c|c|c|c|c|c|c|c|c|c|c|}
\hline$t$ & \multicolumn{10}{c|}{$\mathrm{r}$} \\
\cline { 2 - 11 } & 0 & 0.1 & 0.2 & 0.3 & 0.4 & 0.5 & 0.6 & 0.7 & 0.8 & 0.9 & 1 \\
\hline 1.1 & 2.274208 & 2.350015 & 2.425822 & 2.501629 & 2.577436 & 2.653243 & 2.729050 & 2.804857 & 2.880664 & 2.956471 & 3.032277 \\
1.2 & 2.577355 & 2.663267 & 2.749179 & 2.835091 & 2.921003 & 3.006915 & 3.092826 & 3.178738 & 3.264650 & 3.350562 & 3.436474 \\
1.3 & 2.955267 & 3.053776 & 3.152285 & 3.250794 & 3.349303 & 3.447812 & 3.546321 & 3.644830 & 3.743339 & 3.841848 & 3.940357 \\
1.4 & 3.415807 & 3.529668 & 3.643528 & 3.757388 & 3.871249 & 3.985109 & 4.098969 & 4.212829 & 4.326690 & 4.440550 & 4.554410 \\
1.5 & 3.967666 & 4.099921 & 4.232177 & 4.364432 & 4.496688 & 4.628943 & 4.761199 & 4.893454 & 5.025710 & 5.157966 & 5.290221 \\
1.6 & 4.578278 & 4.730887 & 4.883496 & 5.036106 & 5.188715 & 5.341324 & 5.493934 & 5.646543 & 5.799152 & 5.951761 & 6.104371 \\
1.7 & 5.210226 & 5.383900 & 5.557575 & 5.731249 & 5.904923 & 6.078597 & 6.252271 & 6.425946 & 6.599620 & 6.773294 & 6.946968 \\
1.8 & 5.865754 & 6.061279 & 6.256805 & 6.452330 & 6.647855 & 6.843380 & 7.038905 & 7.234430 & 7.429956 & 7.625481 & 7.821006 \\
1.9 & 6.547342 & 6.765587 & 6.983832 & 7.202077 & 7.420321 & 7.638566 & 7.856811 & 8.075056 & 8.293300 & 8.511545 & 8.729790 \\
2.0 & 7.257731 & 7.499655 & 7.741580 & 7.983504 & 8.225429 & 8.467353 & 8.709277 & 8.951202 & 9.193126 & 9.435050 & 9.676975 \\
\hline
\end{tabular}

Table 1: The approximation solution by RK Nystrom method to the $\operatorname{IVP}(15)-\underline{x}(t ; r)$

\begin{tabular}{|c|c|c|c|c|c|c|c|c|c|c|c|}
\hline \multirow[t]{2}{*}{$t$} & \multicolumn{11}{|c|}{ r } \\
\hline & 0 & 0.1 & 0.2 & 0.3 & 0.4 & 0.5 & 0.6 & 0.7 & 0.8 & 0.9 & 1 \\
\hline 1.1 & 3.411312 & 3.373409 & 3.335505 & 3.297602 & 3.259698 & 3.221795 & 3.183891 & 3.145988 & 3.108084 & 3.070181 & 3.032277 \\
\hline 1.2 & 3.866033 & 3.823077 & 3.780121 & 3.737165 & 3.694209 & 3.651253 & 3.608298 & 3.565342 & 3.522386 & 3.479430 & 3.436474 \\
\hline 1.3 & 4.432901 & 4.383647 & 4.334392 & 4.285138 & 4.235883 & 4.186629 & 4.137375 & 4.088120 & 4.038866 & 3.989611 & 3.940357 \\
\hline 1.4 & 5.123711 & 5.066781 & 5.009851 & 4.952921 & 4.895991 & 4.839061 & 4.782131 & 4.725201 & 4.668270 & 4.611340 & 4.554410 \\
\hline 1.5 & 5.951499 & 5.885371 & 5.819243 & 5.753115 & 5.686988 & 5.620860 & 5.554732 & 5.488604 & 5.422477 & 5.356349 & 5.290221 \\
\hline 1.6 & 6.867417 & 6.791112 & 6.714808 & 6.638503 & 6.562199 & 6.485894 & 6.409589 & 6.333285 & 6.256980 & 6.180675 & 6.104371 \\
\hline 1.7 & 7.815339 & 7.728502 & 7.641665 & 7.554828 & 7.467991 & 7.381154 & 7.294317 & 7.207480 & 7.120643 & 7.033805 & 6.946968 \\
\hline 1.8 & 8.798632 & 8.700869 & 8.603107 & 8.505344 & 8.407581 & 8.309819 & 8.212056 & 8.114294 & 8.016531 & 7.918768 & 7.821006 \\
\hline 1.9 & 9.821014 & 9.711891 & 9.602769 & 9.493647 & 9.384524 & 9.275402 & 9.166280 & 9.057157 & 8.948035 & 8.838912 & 8.729790 \\
\hline 2.0 & 10.88659 & 10.76563 & 10.64467 & 10.52371 & 10.40274 & 10.28178 & 10.16082 & 10.03986 & 9.918899 & 9.797937 & 9.676975 \\
\hline
\end{tabular}

Table 2: The approximation solution by RK Nystrom method to the IVP(15) - $\bar{x}(t ; r)$

Then

$y_{2.0 ; r}=c_{2.0} y_{1}(1.0 ; r)$,

$=\left[c_{2,0}(0.75+0.25 r)\left(c_{1.0}\right)^{10}, c_{2,0}(1.125-0.125 r)\left(c_{1.0}\right)^{10}\right], 0 \leq r \leq 1$.

Since the exact solution of (5.4) for $t \in[1,1.5]$ is $x(t ; r)=x(1 ; r)\left(3 e^{t-1}-2 t\right), 0 \leq r \leq 1, x(1.5 ; r)=x(1 ; r)(3 \sqrt{e}-3)$, $0 \leq r \leq 1$. Then $x(1.5 ; r)$ is approximately 5.29022058 and $y_{1,1}$ is approximately 5.29022158. Since the exact solution of $(5.4)$ for $t \in[1.5,2]$ is $x(t ; r)=$ $x(1 ; r)\left(2 t-2+e^{t-1.5}(3 \sqrt{e}-4)\right), 0 \leq r \leq 1$.

Therefore $x(2.0 ; r)=x(1 ; r)(2+3 e-4 \sqrt{e})$. Then $x(2.0 ; r)$ is approximately 9.676975672 and $y_{1}(2.0 ; 1)$ is approximately 9.676975795 . The approximate solution by fifth order Runge Kutta Nystrom method is plotted at $t \in[0,2]($ see Table 1-4 and Figure 3.1). The exact and approximate solution by fifth order Runge Kutta Nystrom method is plotted at $t=2$. (see Table 1-4 and Figure 3.2).

Example 5.2. Next consider the following hybrid fuzzy IVP,

$$
\left\{\begin{array}{l}
x^{\prime}(t)=x(t)+m(t) \lambda_{k} x\left(t_{k}\right), t \in\left[t_{k}, t_{k+1}\right], t_{k}=k, k=0,1,2,3, \ldots, \\
x(t ; r)=\left[(0.75+0.25 r) e^{t},(1.125-0.125 r) e^{t}\right], \quad 0 \leq r \leq 1,
\end{array}\right.
$$

where

$$
m(t)=|\sin (\pi t)|, \quad k=0,1,2, \ldots,
$$

\begin{tabular}{|c|c|c|c|c|c|c|c|c|c|c|c|}
\hline \multirow[t]{2}{*}{$t$} & \multicolumn{11}{|c|}{$\mathrm{r}$} \\
\hline & 0 & 0.1 & 0.2 & 0.3 & 0.4 & 0.5 & 0.6 & 0.7 & 0.8 & 0.9 & 1 \\
\hline 1.1 & 2.274208 & 2.350015 & 2.425822 & 2.501629 & 2.577436 & 2.653243 & 2.729050 & 2.804857 & 2.880664 & 2.956471 & 3.032278 \\
\hline 1.2 & 2.577355 & 2.663267 & 2.749179 & 2.835091 & 2.921003 & 3.006915 & 3.092826 & 3.178738 & 3.264650 & 3.350562 & 3.436474 \\
\hline 1.3 & 2.955267 & 3.053776 & 3.152285 & 3.250794 & 3.349303 & 3.447812 & 3.546321 & 3.644830 & 3.743339 & 3.841848 & 3.940357 \\
\hline 1.4 & 3.415808 & 3.529668 & 3.643528 & 3.757388 & 3.871249 & 3.985109 & 4.098969 & 4.212829 & 4.326690 & 4.440550 & 4.554410 \\
\hline 1.5 & 3.967666 & 4.099921 & 4.232177 & 4.364432 & 4.496688 & 4.628944 & 4.761199 & 4.893455 & 5.025710 & 5.157966 & 5.290221 \\
\hline 1.6 & 4.578278 & 4.730887 & 4.883497 & 5.036106 & 5.188715 & 5.341324 & 5.493934 & 5.646543 & 5.799152 & 5.951762 & 6.104371 \\
\hline 1.7 & 5.210226 & 5.383901 & 5.557575 & 5.731249 & 5.904923 & 6.078597 & 6.252272 & 6.425946 & 6.599620 & 6.773294 & 6.946969 \\
\hline 1.8 & 5.865754 & 6.061280 & 6.256805 & 6.452330 & 6.647855 & 6.843380 & 7.038905 & 7.234431 & 7.429956 & 7.625481 & 7.821006 \\
\hline 1.9 & 6.547343 & 6.765587 & 6.983832 & 7.202077 & 7.420322 & 7.638566 & 7.856811 & 8.075056 & 8.293301 & 8.511545 & 8.729790 \\
\hline 2.0 & 7.257731 & 7.499656 & 7.741580 & 7.983504 & 8.225429 & 8.467353 & 8.709278 & 8.951202 & 9.193126 & 9.435051 & 9.676975 \\
\hline
\end{tabular}

Table 3: The Exact solution to the $\operatorname{IVP}(15)-\underline{x}(t ; r)$ 


\begin{tabular}{|c|c|c|c|c|c|c|c|c|c|c|c|}
\hline$t$ & \multicolumn{10}{c|}{$\mathrm{r}$} \\
\cline { 2 - 11 } & 0 & 0.1 & 0.2 & 0.3 & 0.4 & 0.5 & 0.6 & 0.7 & 0.8 & 0.9 & 1 \\
\hline 1.1 & 3.411312 & 3.373409 & 3.335505 & 3.297602 & 3.259698 & 3.221795 & 3.183891 & 3.145988 & 3.108084 & 3.070181 & 3.032278 \\
1.2 & 3.866033 & 3.823077 & 3.780121 & 3.737165 & 3.694209 & 3.651254 & 3.608298 & 3.565342 & 3.522386 & 3.479430 & 3.436474 \\
1.3 & 4.432901 & 4.383647 & 4.334392 & 4.285138 & 4.235884 & 4.186629 & 4.137375 & 4.088120 & 4.038866 & 3.989611 & 3.940357 \\
1.4 & 5.123712 & 5.066781 & 5.009851 & 4.952921 & 4.895991 & 4.839061 & 4.782131 & 4.725201 & 4.668271 & 4.611340 & 4.554410 \\
1.5 & 5.951499 & 5.885371 & 5.819243 & 5.753116 & 5.686988 & 5.620860 & 5.554732 & 5.488605 & 5.422477 & 5.356349 & 5.290221 \\
1.6 & 6.867417 & 6.791113 & 6.714808 & 6.638503 & 6.562199 & 6.485894 & 6.409589 & 6.333285 & 6.256980 & 6.180676 & 6.104371 \\
1.7 & 7.815340 & 7.728503 & 7.641665 & 7.554828 & 7.467991 & 7.381154 & 7.294317 & 7.207480 & 7.120643 & 7.033806 & 6.946969 \\
1.8 & 8.798632 & 8.700869 & 8.603107 & 8.505344 & 8.407582 & 8.309819 & 8.212056 & 8.114294 & 8.016531 & 7.918769 & 7.821006 \\
1.9 & 9.821014 & 9.711892 & 9.602769 & 9.493647 & 9.384525 & 9.275402 & 9.166280 & 9.057157 & 8.948035 & 8.838913 & 8.729790 \\
2.0 & 10.88659 & 10.76563 & 10.64467 & 10.52371 & 10.40274 & 10.28178 & 10.16082 & 10.03986 & 9.918900 & 9.797937 & 9.676975 \\
\hline
\end{tabular}

Table 4: The Exact solution to the $\operatorname{IVP}(15)-\bar{x}(t ; r)$

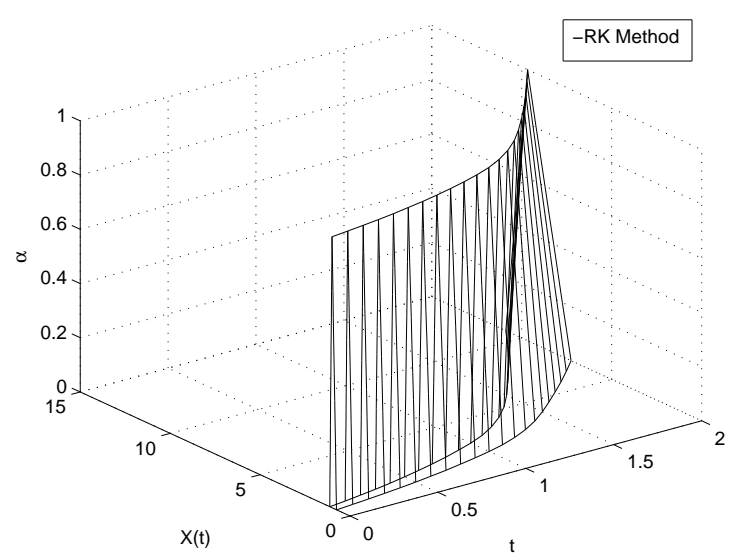

Figure 5.1: (for $h=0.1$ )

$$
\lambda_{k}(\mu)= \begin{cases}\hat{0}, & \text { if } k=0 \\ \mu, & \text { if } k \in\{1,2, \ldots\}\end{cases}
$$

Then $x(t)+m(t) \lambda_{k}\left(x\left(t_{k}\right)\right.$ is continuous function of $t, x$ and $\lambda_{k}\left(x\left(t_{k}\right)\right.$. Therefore by Example 6.1 of Kaleva [6], for each $k=0,1,2, \ldots$, the fuzzy IVP

$$
\left\{\begin{array}{l}
x^{\prime}(t)=x(t)+m(t) \lambda_{k}\left(x\left(t_{k}\right), \quad t \in\left[t_{k}, t_{k+1}\right], t_{k}=k\right. \\
x\left(t_{k}\right)=x_{t_{k}}
\end{array}\right.
$$

has a unique solution on $\left[t_{k}, t_{k+1}\right]$. To numerically solve the hybrid fuzzy IVP (36) we will apply the Runge-Kutta Method of order five for hybrid fuzzy differential equations with $N=10$.

To obtain $y_{1,1}(r)$,

$C_{1}=125 h+\frac{200}{3} h^{2}+20 h^{3}$,

$C_{2}=23 h+24 h^{2}+12 h^{3}+\frac{16}{5} h^{4}+\frac{8}{5} h^{5}$,

$$
\begin{aligned}
\underline{y}(1.1 ; r)= & \underline{y}(1.0 ; r) c_{0,1}+\frac{1}{192}\left[C_{1} \sin \frac{\pi}{25}+125 h \sin \frac{2 \pi}{25}\right. \\
& \left.-81 h \sin \frac{\pi}{15}+\frac{16}{3} h^{2} \sin \frac{\pi}{10}+\frac{24}{5} h^{4} \sin \frac{\pi}{30}\right] \underline{y}(1.0 ; r), \\
\bar{y}(1.1 ; r)= & \bar{y}(1.0 ; r) c_{0,1}+\frac{1}{192}\left[C_{1} \sin \frac{\pi}{25}+125 h \sin \frac{2 \pi}{25}\right. \\
& \left.-81 h \sin \frac{\pi}{15}+\frac{16}{3} h^{2} \sin \frac{\pi}{10}+\frac{24}{5} h^{4} \sin \frac{\pi}{30}\right] \bar{y}(1.0 ; r),
\end{aligned}
$$

Then for $i=1,2,3, \ldots, 10$. 


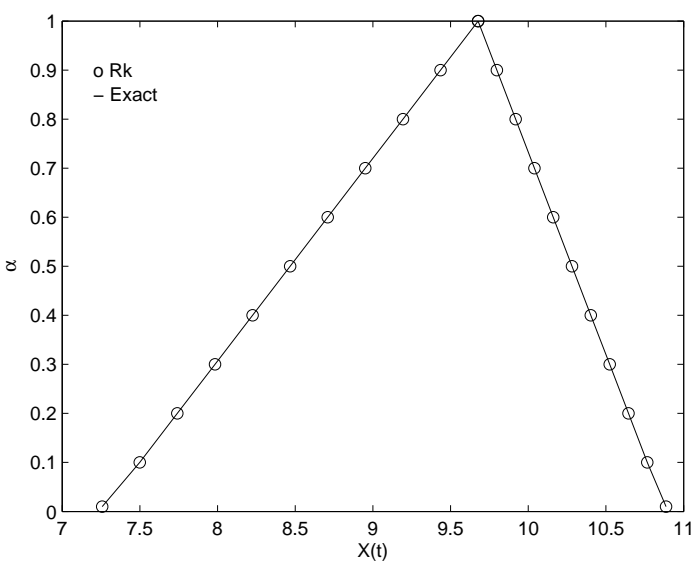

Figure 5.2: (for $h=0.1$ )

$$
\begin{aligned}
\underline{y}\left(1+\frac{i}{10} ; r\right) & =\underline{y}\left(1+\frac{i-1}{10} ; r\right)+\frac{1}{192}\left[C_{1} \sin \frac{(5 i-3) \pi}{50}+C_{2} \sin \frac{(i-1) \pi}{10}\right. \\
& -81 h \sin \frac{(3 i-1) \pi}{30}+125 h \sin \frac{(5 i-1) \pi}{50}+\frac{16}{3} h^{2} \sin \frac{\pi i}{10} \\
+ & \left.\frac{24}{5} h^{4} \sin \frac{(3 i-2)) \pi}{30}\right] \underline{y}(1.0 ; r), \\
\bar{y}\left(1+\frac{i}{10} ; r\right) & =\bar{y}\left(1+\frac{i-1}{10} ; r\right)+\frac{1}{192}\left[C_{1} \sin \frac{(5 i-3) \pi}{50}+C_{2} \sin \frac{(i-1) \pi}{10}\right. \\
- & 81 h \sin \frac{(3 i-1) \pi}{30}+125 h \sin \frac{(5 i-1) \pi}{50}+\frac{16}{3} h^{2} \sin \frac{\pi i}{10} \\
+ & \left.\frac{24}{5} h^{4} \sin \frac{(3 i-2)) \pi}{30}\right] \bar{y}(1.0 ; r) .
\end{aligned}
$$

Let

$$
\begin{aligned}
c_{2,0}= & \left(c_{0,1}\right)^{10}+\sum_{k=1}^{10}\left(c_{0,1}\right)^{10-k} \frac{1}{192}\left[C_{1} \sin \frac{(5 k-3) \pi}{50}+C_{2} \sin \frac{(k-1) \pi}{10}-81 h \sin \frac{(3 k-1) \pi}{30}\right. \\
& \left.+125 h \sin \frac{(5 k-1) \pi}{50}+\frac{16}{3} h^{2} \sin \frac{\pi k}{10}+\frac{24}{5} h^{4} \sin \frac{(3 k-2)) \pi}{30}\right],
\end{aligned}
$$

Then

$y_{2.0 ; r}=c_{2.0} y_{1}(1.0 ; r)$,

$$
=\left[c_{2,0}(0.75+0.25 r)\left(c_{1.0}\right)^{10}, c_{2,0}(1.125-0.125 r)\left(c_{1.0}\right)^{10}\right], 0 \leq r \leq 1 .
$$

for $t \in[1,2]$, the exact solution of (5.7) satisfies

$$
\begin{aligned}
& \underline{x}(t ; r)=\underline{x}(1 ; r) \frac{\pi \cos (\pi t)+\sin (\pi t)}{\pi^{2}+1}+\frac{e^{t}}{e} \underline{x}(1 ; r)\left(1+\frac{\pi}{\pi^{2}+1}\right), \\
& \bar{x}(t ; r)=\bar{x}(1 ; r) \frac{\pi \cos (\pi t)+\sin (\pi t)}{\pi^{2}+1}+\frac{e^{t}}{e} \bar{x}(1 ; r)\left(1+\frac{\pi}{\pi^{2}+1}\right) .
\end{aligned}
$$

Therefore

$$
\begin{aligned}
& x(1 ; r)=[(0.75+0.25 r) e,(1.125-0.125 r) e], \\
& x(2 ; r)=\left(\frac{\pi}{\pi^{2}+1}+e\left(1+\frac{\pi}{\pi^{2}+1}\right)\right) x(1 ; r) .
\end{aligned}
$$

Then $x(2.0 ; 1)$ is approximately 10.31033432 where as $y_{1}(2.0 ; 1)$ is approximately 10.31033708 . The approximate solution by fifth order Runge Kutta Nystrom method is plotted at $t \in[0,2]$ (see Table 5-8 and Figure 3.3). The exact and approximate solution by fifth order Runge Kutta Nystrom method is plotted at $t=2$. (see Table 5-8 and Figure 5.4).

\section{Conclusion}

In this paper we have discussed hybrid fuzzy differential systems and applied fifth order Runge-Kutta Nystorm method. In the proposed method is convergent to order $O\left(h^{6}\right)$. 


\begin{tabular}{|c|c|c|c|c|c|c|c|c|c|c|c|}
\hline$t$ & \multicolumn{10}{c|}{$\mathrm{r}$} \\
\cline { 2 - 11 } & 0 & 0.1 & 0.2 & 0.3 & 0.4 & 0.5 & 0.6 & 0.7 & 0.8 & 0.9 & 1 \\
\hline 1.1 & 2.285975 & 2.362174 & 2.438373 & 2.514572 & 2.590772 & 2.666971 & 2.743170 & 2.819369 & 2.895568 & 2.971767 & 3.047967 \\
1.2 & 2.622836 & 2.710264 & 2.797691 & 2.885119 & 2.972547 & 3.059975 & 3.147403 & 3.234831 & 3.322259 & 3.409687 & 3.497114 \\
1.3 & 3.049276 & 3.150919 & 3.252561 & 3.354204 & 3.455846 & 3.557489 & 3.659131 & 3.760774 & 3.862416 & 3.964059 & 4.065701 \\
1.4 & 3.559976 & 3.678642 & 3.797307 & 3.915973 & 4.034639 & 4.153305 & 4.271971 & 4.390637 & 4.509303 & 4.627968 & 4.746634 \\
1.5 & 4.145197 & 4.283371 & 4.421544 & 4.559717 & 4.697890 & 4.836064 & 4.974237 & 5.112410 & 5.250583 & 5.388757 & 5.526930 \\
1.6 & 4.792142 & 4.951881 & 5.111619 & 5.271357 & 5.431095 & 5.590833 & 5.750571 & 5.910309 & 6.070047 & 6.229785 & 6.389523 \\
1.7 & 5.486649 & 5.669538 & 5.852426 & 6.035314 & 6.218203 & 6.401091 & 6.583979 & 6.766868 & 6.949756 & 7.132644 & 7.315533 \\
1.8 & 6.215071 & 6.422240 & 6.629409 & 6.836578 & 7.043747 & 7.250916 & 7.458086 & 7.665255 & 7.872424 & 8.079593 & 8.286762 \\
1.9 & 6.966156 & 7.198362 & 7.430567 & 7.662772 & 7.894977 & 8.127183 & 8.359388 & 8.591593 & 8.823798 & 9.056004 & 9.288209 \\
2.0 & 7.732750 & 7.990509 & 8.248267 & 8.506025 & 8.763784 & 9.021542 & 9.279300 & 9.53705 & 9.794817 & 10.05257 & 10.31033 \\
\hline
\end{tabular}

Table 5: The approximation solution by RK Nystrom method to the IVP(18) - $\underline{x}(t ; r)$

\begin{tabular}{|c|c|c|c|c|c|c|c|c|c|c|c|}
\hline \multirow[t]{2}{*}{$t$} & \multicolumn{11}{|c|}{$\mathrm{r}$} \\
\hline & 0 & 0.1 & 0.2 & 0.3 & 0.4 & 0.5 & 0.6 & 0.7 & 0.8 & 0.9 & 1 \\
\hline 1.1 & 3.428962 & 3.390863 & 3.352763 & 3.314664 & 3.276564 & 3.238465 & 3.200365 & 3.162265 & 3.124166 & 3.086066 & 3.047967 \\
\hline 1.2 & 3.934254 & 3.890540 & 3.846826 & 3.803112 & 3.759398 & 3.715684 & 3.671970 & 3.628256 & 3.584542 & 3.540828 & 3.497114 \\
\hline 1.3 & 4.573914 & 4.523093 & 4.472272 & 4.421450 & 4.370629 & 4.319808 & 4.268987 & 4.218165 & 4.167344 & 4.116523 & 4.065701 \\
\hline 1.4 & 5.339964 & 5.280631 & 5.221298 & 5.161965 & 5.102632 & 5.043299 & 4.983966 & 4.924633 & 4.865300 & 4.805967 & 4.746634 \\
\hline 1.5 & 6.217796 & 6.148710 & 6.079623 & 6.010536 & 5.941450 & 5.872363 & 5.803276 & 5.734190 & 5.665103 & 5.596017 & 5.526930 \\
\hline 1.6 & 7.188214 & 7.108345 & 7.028476 & 6.948607 & 6.868738 & 6.788869 & 6.709000 & 6.629131 & 6.549261 & 6.469392 & 6.389523 \\
\hline 1.7 & 8.229974 & 8.138530 & 8.047086 & 7.955642 & 7.864197 & 7.772753 & 7.681309 & 7.589865 & 7.498421 & 7.406977 & 7.315533 \\
\hline 1.8 & 9.322607 & 9.219023 & 9.115438 & 9.011853 & 8.908269 & 8.804684 & 8.701100 & 8.597515 & 8.493931 & 8.390346 & 8.286762 \\
\hline 1.9 & 10.44923 & 10.33313 & 10.21703 & 10.10092 & 9.984824 & 9.868722 & 9.752619 & 9.636517 & 9.520414 & 9.404311 & 9.288209 \\
\hline 2.0 & 11.59912 & 11.47024 & 11.34136 & 11.21248 & 11.08360 & 10.95473 & 10.82585 & 10.69697 & 10.56809 & 10.43921 & 10.31033 \\
\hline
\end{tabular}

Table 6: The approximation solution by RK Nystrom method to the IVP(18)- $\bar{x}(t ; r)$ for

\begin{tabular}{|c|c|c|c|c|c|c|c|c|c|c|c|}
\hline \multirow[t]{2}{*}{$t$} & \multicolumn{11}{|c|}{$\mathrm{r}$} \\
\hline & 0 & 0.1 & 0.2 & 0.3 & 0.4 & 0.5 & 0.6 & 0.7 & 0.8 & 0.9 & 1 \\
\hline 1.1 & 2.285975 & 2.362174 & 2.438373 & 2.514572 & 2.590772 & 2.666971 & 2.743170 & 2.819369 & 2.895568 & 2.971767 & 3.047967 \\
\hline 1.2 & 2.622836 & 2.710264 & 2.797691 & 2.885119 & 2.972547 & 3.059975 & 3.147403 & 3.234831 & 3.322259 & 3.409687 & 3.497114 \\
\hline 1.3 & 3.049276 & 3.150919 & 3.252561 & 3.354204 & 3.455846 & 3.557489 & 3.659131 & 3.760774 & 3.862416 & 3.964059 & 4.065702 \\
\hline 1.4 & 3.559976 & 3.678642 & 3.797307 & 3.915973 & 4.034639 & 4.153305 & 4.271971 & 4.390637 & 4.509303 & 4.627968 & 4.746634 \\
\hline 1.5 & 4.145197 & 4.283371 & 4.421544 & 4.559717 & 4.697890 & 4.836064 & 4.974237 & 5.112410 & 5.250583 & 5.388757 & 5.526930 \\
\hline 1.6 & 4.792142 & 4.951880 & 5.111619 & 5.271357 & 5.431095 & 5.590833 & 5.750571 & 5.910309 & 6.070047 & 6.229785 & 6.389523 \\
\hline 1.7 & 5.486649 & 5.669538 & 5.852426 & 6.035314 & 6.218203 & 6.401091 & 6.583979 & 6.766867 & 6.949756 & 7.132644 & 7.315532 \\
\hline 1.8 & 6.215071 & 6.422240 & 6.629409 & 6.836578 & 7.043747 & 7.250916 & 7.458085 & 7.665255 & 7.872424 & 8.079593 & 8.286762 \\
\hline 1.9 & 6.966156 & 7.198362 & 7.430567 & 7.662772 & 7.894977 & 8.127183 & 8.359388 & 8.591593 & 8.823798 & 9.056003 & 9.288209 \\
\hline 2.0 & 7.732750 & 7.990509 & 8.248267 & 8.506025 & 8.763784 & 9.021542 & 9.279300 & 9.537059 & 9.794817 & 10.05257 & 10.31033 \\
\hline
\end{tabular}

Table 7: The exact solution to the IVP(18) - $\underline{x}(t ; r)$

\begin{tabular}{|c|c|c|c|c|c|c|c|c|c|c|c|}
\hline \multirow[t]{2}{*}{$t$} & \multicolumn{11}{|c|}{$\mathrm{r}$} \\
\hline & 0 & 0.1 & 0.2 & 0.3 & 0.4 & 0.5 & 0.6 & 0.7 & 0.8 & 0.9 & 1 \\
\hline 1.1 & 3.428963 & 3.390863 & 3.352763 & 3.314664 & 3.276564 & 3.238465 & 3.200365 & 3.162265 & 3.124166 & 3.086066 & 3.047967 \\
\hline 1.2 & 3.934254 & 3.890540 & 3.846826 & 3.803112 & 3.759398 & 3.715684 & 3.671970 & 3.628256 & 3.584542 & 3.540828 & 3.497114 \\
\hline 1.3 & 4.573914 & 4.523093 & 4.472272 & 4.421450 & 4.370629 & 4.319808 & 4.268987 & 4.218165 & 4.167344 & 4.116523 & 4.065702 \\
\hline 1.4 & 5.339964 & 5.280631 & 5.221298 & 5.161965 & 5.102632 & 5.043299 & 4.983966 & 4.924633 & 4.865300 & 4.805967 & 4.746634 \\
\hline 1.5 & 6.217796 & 6.148710 & 6.079623 & 6.010536 & 5.941450 & 5.872363 & 5.803276 & 5.734190 & 5.665103 & 5.596017 & 5.526930 \\
\hline 1.6 & 7.188214 & 7.108345 & 7.028476 & 6.948607 & 6.868738 & 6.788869 & 6.709000 & 6.629131 & 6.549261 & 6.469392 & 6.389523 \\
\hline 1.7 & 8.229974 & 8.138530 & 8.047086 & 7.955642 & 7.864197 & 7.772753 & 7.681309 & 7.589865 & 7.498421 & 7.406977 & 7.315532 \\
\hline 1.8 & 9.322607 & 9.219022 & 9.115438 & 9.011853 & 8.908269 & 8.804684 & 8.701100 & 8.597515 & 8.493931 & 8.390346 & 8.286762 \\
\hline 1.9 & 10.44923 & 10.33313 & 10.21703 & 10.10092 & 9.984824 & 9.868722 & 9.752619 & 9.636516 & 9.520414 & 9.404311 & 9.288209 \\
\hline 2.0 & 11.59912 & 11.47024 & 11.34136 & 11.21248 & 11.08360 & 10.95473 & 10.82585 & 10.69697 & 10.56809 & 10.43921 & 10.31033 \\
\hline
\end{tabular}




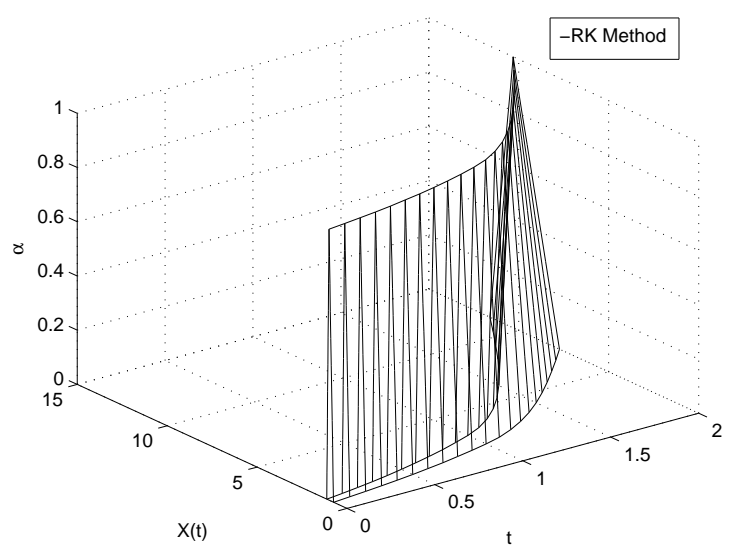

Figure 5.3: (for $h=0.1$ )

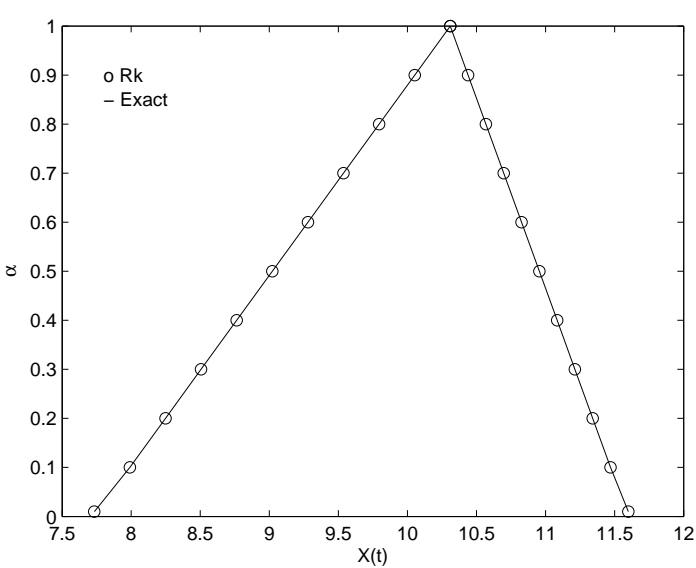

Figure 5.4: (for $h=0.1$ )

\section{References}

[1] S. L. Chang and A. Zadeh, On Fuzzy Mapping and Control, IEEEE Trans. Systems Man Cybernet 2 (1972), 30-34.

[2] D. Dubois and H.Prade, Towards Fuzzy Differential Calculus: Part 3, Differentiation, Fuzzy Sets and System, 8 (1982,) 225-233.

[3] M. L. Puri, D.A. Ralescu, Differentials of fuzzy functions, J. Math. Anal. Appl., 91 (1983), 321-325.

[4] R. Goetschel, W.Voxman, Elementary fuzzy calculus, Fuzzy Sets and Systems, 18 (1986), 31-43.

[5] A. Kandel, W.J. Byatt, Fuzzy Differential Equations, in Proceedings of the International Conference on Cybernetics and Society, Tokyo, (1978) $1213-1216$

[6] O. Kaleva, Fuzzy Differential Equations, Fuzzy Sets and Systems, 24 (1987), 301-317.

[7] O. Kaleva, The Cauchy problem for Fuzzy Differential Equations, Fuzzy Sets and Systems, 35 (1990), $389-396$.

[8] S. Seikkala, On the fuzzy initial value problem, Fuzzy Sets and Systems, 24 (1987), 319-330.

[9] O. He, W. Yi, On fuzzy differential Equations, Fuzzy Sets Systems, 24 (1989), 321-325.

[10] P. Kloedan, Remarks on Piano-like theorems for fuzzy differential Equations, Fuzzy Sets and Systems, 44 (1991), 161-164.

[11] S. Pederson, M. Sambandham, Numerical solution to Hybrid fuzzy systems, Mathematical and Computer Modelling, 45 (2007), $1133-1144$.

[12] L. J. Jowers, J. J. Buckley K. D. Reilly, Simulating continuous fuzzy systems, Inform. Sci., 177 (2007), $436-448$.

[13] T. Jayakumar, K. Kanakarajan, Numerical Solution for Hybrid Fuzzy System by Improved Euler Method, International Journal of Applied Mathematical Science, 38 (2012), 1847-1862.

[14] T. Jayakumar, K. Kanakarajan, Numerical Solution for Hybrid Fuzzy System by Runge Kutta Verner Method, Far East Journal of Applied Mathematics, 86 (2014), 93-115.

[15] T. Jayakumar, K. Kanakarajan, Numerical Solution for Hybrid Fuzzy System by Runge Kutta Method of order five, Applied Mathematical Science, 6 (2012), 69-72.

[16] K. Kanakarajan, M.Sambath, Numerical Solution for hybrid fuzzy differential equations by improved predictor-corrector method, Nonlinear Studies, 19 (2012), 171-185.

[17] M. Sambandham, Perturbed Lyapunov-like functions and Hybrid Fuzzy Differential Equations, Int. J. Hybrid Syst., 2 (2002) 23-34.

[18] C. X. Wu, M. Ma, Embedding problem of fuzzy number space Part I, Fuzzy Sets Syst.,44 (1991) 33-38.

[19] J. J.Bukley, T. Feuring, Fuzzy Differential Equations, Fuzzy Sets Syst., 110 (200) 43-54. 